DOI: 10.12731/2227-930X-2021-11-3-89-98

УДК 004.94

\title{
ОПТИМИЗАЦИЯ СТРУКТУРЫ И ФУНКЦИОНИРОВАНИЯ КОМПЛЕКСНОГО ТРАНСПОРТНОГО ПРЕДПРИЯТИЯ
}

\author{
Львович Я.Е., Преображенский А.П., \\ Преображенский Ю.П.
}

В статье рассматривается задача, связанная с проведением оптимизации структуры и функционирования комплексного транспортного предприятия. Показаны компоненты алгоритмического обеспечения.

Ключевые слова: система перевозок; структура; предприятие; управление

\section{OPTIMIZATION OF THE STRUCTURE AND FUNCTIONING OF THE INTEGRATED TRANSPORTATION ENTERPRISE}

\section{Lvovich Ya.E., Preobrazhenskiy A.P., Preobrazhenskiy Yu.P.}

The paper deals with the problem associated with the optimization of the structure and functioning of an integrated transport company. The components of the algorithmic support are shown.

Ключевые слова: transportation system; structure; enterprise; control

\section{Введение}

Систему массового обслуживания (CMO) можно рассматривать как основу для моделирования комплексного транспортного предприятия. Тогда будет создан подход, направленный на управление транспортным предприятием [1]. В нем используются $m=\overline{1, M}$ 
входных потоков. Какую информацию на их основе можно получить? Они показывают потребность пользователей транспорта в $m$-м виде транспортного обслуживания. Потоки преобразуются. Это происходит на базе соответствующей технологической структуры. Внутри транспортного предприятия можно наблюдать приоритетность по обслуживанию потоков. С чем это связано? Интенсивности потоков являются переменными [2]. Это, в том числе, не дает возможностей для того, чтобы в ходе исследований опираться на аналитическую модель. Вследствие сложности анализируемой транспортной системы необходимо ориентироваться на комплексированный подход. В нем происходит агрегация модели СМО, а также алгоритмов, направленных на структурную и параметрическую оптимизацию. Модели многоальтернативной оптимизации позволяют решать в транспортных системах структурные задачи. Модели, показывающие связь выходных показателей и параметров транспортных систем, позволяют решать параметрические задачи.

Цель статьи заключается в разработке подхода, связанного с оптимизацией структуры и работы в транспортной системе.

\section{Модель транспортной системы}

Предположим, что функционирует транспортная организация. Если строить ее модель, то в одной из возможных требуется множество потоков пользователей транспортных средств $r_{j}, j=\overline{1, J}$. При этом $J$ - будет множество по все типов потоков пользователей транспортных средств. Кроме того, необходимо учесть множество видов транспортного обслуживания $v_{t}, t=\overline{1, T}$. При этом $T$ - будет множеством по всем видам в транспортном обслуживании [3]. Как описать структуру транспортной организации? Она задается при помощи множества элементов $r_{j}, j=\overline{1, J}, v_{t}, t=\overline{1, T}$ и связей среди них:

$$
S \subset R \times V .
$$

Как в транспортной организации промоделировать структуру? Основываясь на опыте различных исследователей, можно ориентироваться на три ее вида: последовательную, сходящуюся и расходящуюся (рис. 1). 
Есть особенность, связанная с первой структурой. По каждому из каналов существует возможность наблюдения одного входного, а также выходного потока. Выходя из одного звена, поток будет идти к входу другого звена. Иллюстрация последовательной структуры будет такой:

$$
s_{l}=\left(r_{1}, v_{1}, \ldots ; r_{G}, v_{G}\right) .
$$

При этом $G$ - рассматривается как число звеньев, которые будут последовательными, $l=\overline{1, L}$ - анализируется в виде варианта применения последовательной структуры, $L$ - рассматривается в виде общего числа вариантов структур [4].

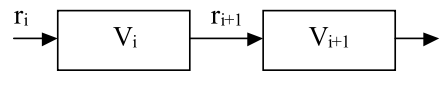

Последовательная структура

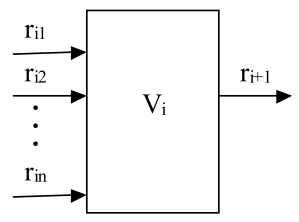

Сходящаяся структура

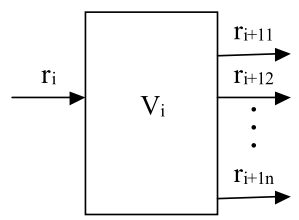

Расходящаяся структура

Рис. 1. Иллюстрация разных видов структур СМО

Если структура сходится, тогда входящих потоков будет множество. При этом будет наблюдаться один из выходящих потоков. Эта структура с точки зрения ее аналитической записи будет представляться на основе списка:

$$
s_{l}=\left(r_{11}, \ldots, r_{1 N_{1}}, v_{1}, \ldots, r_{G 1}, \ldots, r_{G N_{G}}, v_{G}\right) \text {. }
$$

При этом $N_{i}$ - показывает число входных потоков , которые соответствуют $i$-му звену. Если структура рассматривается как рас- 
ходящаяся, тогда с ней будут соотноситься множество выходящих потоков, а также один входящий [5]. Какое подразделение может быть промоделировано при помощи расходящейся структуры в транспортной организации? Например, может быть рассмотрен отдел регистрации пользователей транспортных средств. Кто создает входные и выходные потоки. Первые формируются поступившими пользователями. Вторые формируются пользователями, которые являются распределенными относительно разных подразделений [6]. Каким образом сделать представление расходящейся структуры? Например, так :

$$
s_{l}=\left(r_{1}, v_{1}, r_{21}, \ldots, r_{2 N_{2}}, \ldots ; r_{G-1}, v_{G-1}, r_{G 1}, \ldots, r_{G N_{G}}\right) .
$$

При этом $N_{i}$ - рассматривается в числом выходных потоков, которые соответствуют (i-1)-му звену. Как уже было сказано, рассмотренные три структуры являются базовыми. На основе их комбинирования существуют возможности для получения структур с самыми разными свойствами.

Какие можно отметить особенностей в ходе решения задач структурной и параметрической оптимизации транспортной системы? Среди них существуют такие:

1. Критерии оптимальности бывают множественными, иногда в них наблюдаются противоречия.

2. По всем потокам трудно осуществить процессы предсказаний. Трудности могут оставаться, даже если существует неполная априорная информации. Какие методы могут быть применены в перспективе? Используются вероятностные меры, которые позволяют в транспортной системе учесть неопределенность по внутренним связям.

3. В том числе, из второй особенности вытекает крайне ограниченное число аналитических методик, позволяющих управлять транспортными системами.

Комплексное транспортное предприятие при имитационном моделировании может быть проанализировано при учете того, что структурные и параметрические элементы варьируются [7, 8]. Также схемой выбора будет являться рандомизированной. Су- 
ществуют логические блоки, рассматриваемые в виде структурных элементов транспортных систем. Они учитываются в ходе процессов имитационного моделирования. За счет него происходит определение структуру транспортных видов обеспечения. Откуда можно считывать параметры? Они связаны с временными характеристиками по входным и промежуточным потокам однородных событий.

Транспортное предприятие необходимо стремиться делатьгибким. Структурные его компоновки могут вестись на базе разных вариантов. При этом применяются методы многоальтернативной оптимизации. вводятся Процессы переходов внутри логических блоков описываются с привлечением вероятностных способов. Рандомизированные алгоритмы дают возможности для того, чтобы проводить настройку значений вероятностей. На рис. 2 показаны составляющие алгоритмического обеспечения. Генераторы случайных последовательностей $\Phi_{m}(m=\overline{1, M})$, которые соотнесены с параметрами $x_{j}(j=\overline{1, J})$ позволяют внутри транспортного предприятия получать $m=\overline{1, M}$ потоков однородных событий.

Поток без последействия дают возможности для описания потоков пользователей транспортных средств, они будут простейшими. Интенсивность потока входит в показательную функцию, которая рассматривается в виде плотность распределения интервалов среди поступлений событий.

Среднее время, которое будет между поступлением заявок будет обратно пропорционально величине интенсивности потока. Тогда создается случайная величина, которая будет распределена в рамках экспоненциального закона

$$
\eta=-\frac{1}{\lambda} \lg \xi
$$

При этом $\xi$ рассматривается в виде случайной величины, которая равномерным образом будет распределена внутри интервала $(0,1)$.

Внутри транспортных систем могут наблюдаться также потоки Эрланга. 


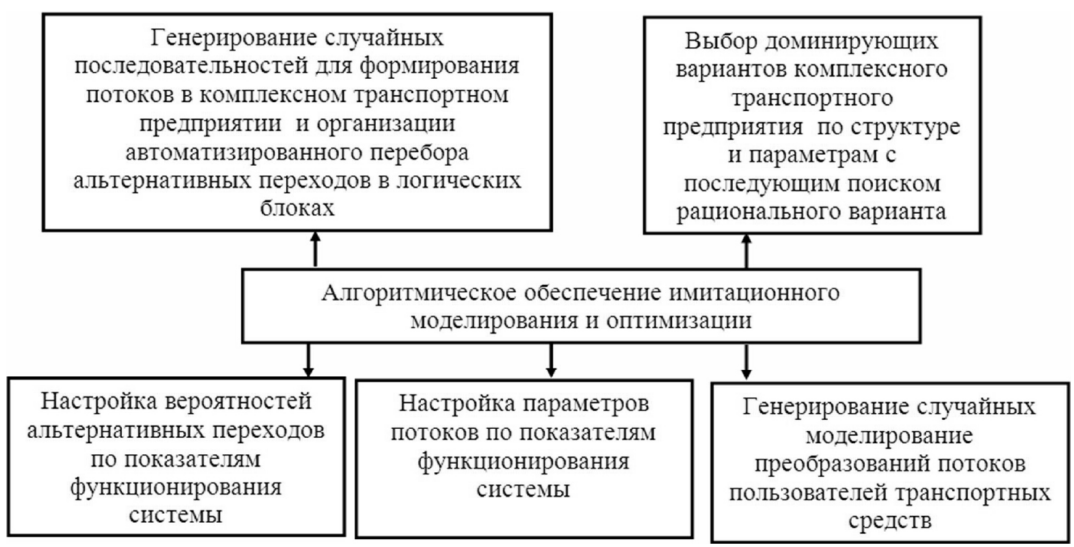

Рис. 2. Компоненты алгоритмического обеспечения имитационного моделирования и оптимизации

То, насколько $m$-й поток участвует при формировании модели преобразования, соотносится с $m$-логическим блоком, который связан с альтернативной переменной $[9,10]$

$Z_{m}=\left\{\begin{array}{l}1, \text { если m-й блок формируетслучайнуюпоследовательность заявок } \\ \text { для модели преобразования; } \\ 0, \text { в противном случае. }\end{array}\right.$

Реализация альтернативных переходов происходит, исходя из вероятности $P_{z m}$ в рамках правила

$$
Z_{m}=\left\{\begin{array}{l}
1, \text { если } \xi \leq P_{z m}, \\
0, \text { если }>P_{z m},
\end{array}\right.
$$

При этом $\xi$ рассматриваются в виде случайных чисел, которые равномерным образом будут распределенными по интервалу $[0,1]$.

По комплексному транспортному предприятию вариант его структуры может быть описан при помощи показателей $F_{i}(i=\overline{1, I})$. При этом $I$ рассматривается в виде общего числа вариантов. Также вводятся переменные $Z_{m}(m=\overline{1, M})$. Введены обозначения $M$ - соответствует общему числу звеньев в структуре транспортного предприятия. Также переменные $x_{j}(j=\overline{1, J})$, при этом $J$ - рассматривается в виде числа входных потоков. Необходимо выполнение зависимости

$$
F_{i}=\Psi\left(Z_{m}, x_{j}\right)
$$


Рандомизированные схемы дают возможности для того, чтобы вероятности $P_{z m} \quad(m=\overline{1, M})$ были настроены. Также ведется определение параметров $x_{j}(j=\overline{1, J})$, соответствующих распределениям $\Phi_{m}(m=\overline{1, M})$. На определенном этапе происходит совмещение с параметрической оптимизацией.

Когда реализуются процедуры, связанные с настройкой, то получаются распределения $P_{z m}, \Phi_{m}$.

Они необходимы для того, чтобы генерировать $n=\overline{1, N}$ вариантов структур транспортных предприятий, а также параметров, связанных с каждым из вариантов показателей. На рис. 3 можно увидеть общую схему, связанную с имитационным моделированием и оптимизацией параметров комплексного транспортного предприятия.

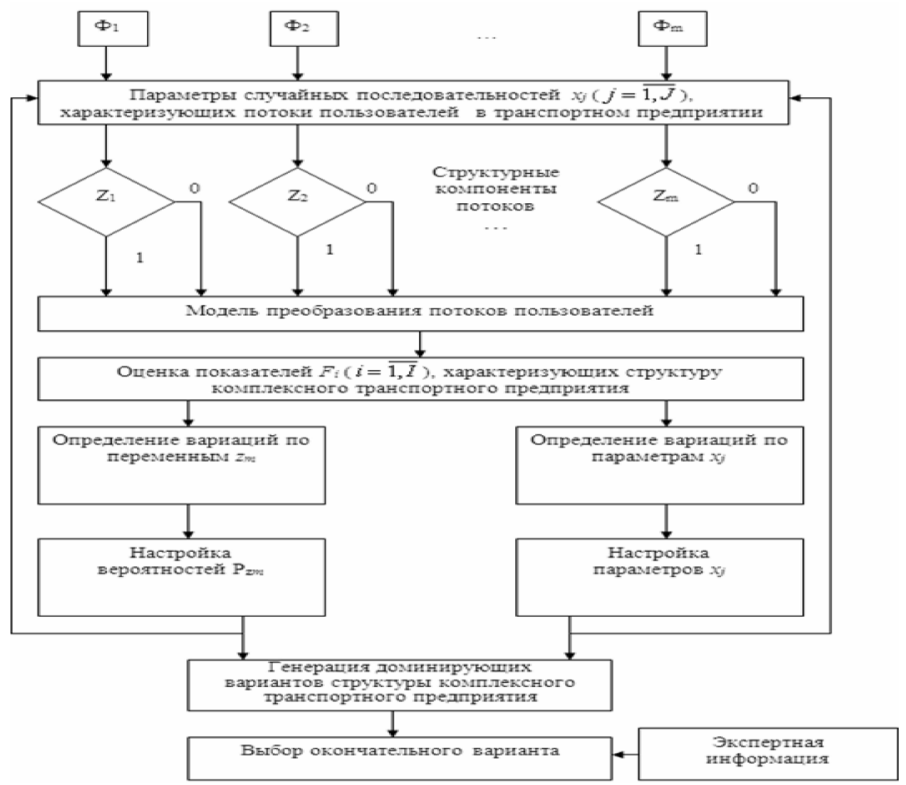

Рис. 3. Иллюстрация схемы имитационного моделирования и оптимизации параметров комплексного транспортного предприятия

\section{Выводы}

В работе рассмотрена задача оптимизации работы транспортных систем. Показаны соответствующие компоненты алгорит- 
мического обеспечения имитационного моделирования и оптимизации.

Информация о конфликте интересов. Авторы заявляют об отсутствии конфликта интересов.

Информация о спонсорстве. Исследование не имело спонсорской поддержки.

\section{Сиисок литературь}

1. Жилина А.А., Кострова В.Н., Преображенский Ю.П. Разработка методики постановки задачи выбора управленческого решения на основе оптимизационного подхода // Моделирование, оптимизация и информационные технологии. 2018. Т. 6, № 1 (20). С. 243-253.

2. Преображенский Ю.П. Информационные технологии, используемые в сфере менеджмента // Вестник Воронежского института высоких технологий. 2018. № 2 (25). С. 43-46.

3. Преображенский Ю.П., Мирошник Д.Н. Анализ методов нечеткого поиска // Вестник Воронежского института высоких технологий. 2018. № 4 (27). С. 82-84.

4. Преображенский Ю.П., Коновалов В.М. О методах создания рекомендательных систем // Вестник Воронежского института высоких технологий. 2019. № 4 (31). С. 75-79.

5. Берман Н.Д., Белов А.М. Общественный транспорт и инновации // International Journal of Advanced Studies. 2019. T. 9, № 2. C. 7-13.

6. Шакиров А.А., Зарипова Р.С. Особенности моделирования логистических систем // International Journal of Advanced Studies. 2019. T. 9, № 4. C. 27-31.

7. Сапожникова С.М. Корпоративное управление в железнодорожном транспорте // International Journal of Advanced Studies. 2019. T. 9, № 4. C. 19-42.

8. Грошев А.Г., Фролов В.Н., Федорков Е.Д. Построение онтологических моделей систем автоматизированного проектирования // Вестник Воронежского института высоких технологий. 2020. № 4 (35). С. 52-56.

9. Львович Э.М., Холодков А.М. Проблемы передачи информации в автоматизированных системах управления // Вестник Воронежского института высоких технологий. 2020. № 3 (34). С. 30-33. 
10. Львович Я.Е., Львович И.Я., Львович Э.М. Проблемы обработки цифровых сигналов в системах передачи информации // Вестник Воронежского института высоких технологий. 2020. № 3 (34). С. 27-29.

\section{References}

1. Zhilina A.A., Kostrova V.N., Preobrazhenskiy Yu.P. Modelirovanie, optimizatsiya i informatsionnye tekhnologii, 2018, vol. 6, no. 1 (20), pp. 243-253.

2. Preobrazhenskiy Yu.P. Vestnik Voronezhskogo instituta vysokikh tekhnologiy, 2018, no. 2 (25), pp. 43-46.

3. Preobrazhenskiy Yu.P., Miroshnik D.N. Vestnik Voronezhskogo instituta vysokikh tekhnologiy, 2018, no. 4 (27), pp. 82-84.

4. Preobrazhenskiy Yu.P., Konovalov V.M. Vestnik Voronezhskogo instituta vysokikh tekhnologiy, 2019, no. 4 (31), pp. $75-79$.

5. Berman N.D., Belov A.M. International Journal of Advanced Studies, 2019, vol. 9, no. 2, pp. 7-13.

6. Shakirov A.A., Zaripova R.S. International Journal of Advanced Studies, 2019, vol. 9, no. 4, pp. 27-31.

7. Sapozhnikova S.M. International Journal of Advanced Studies, 2019, vol. 9, no. 4, pp. 19-42.

8. Groshev A.G., Frolov V.N., Fedorkov E.D. Vestnik Voronezhskogo instituta vysokikh tekhnologiy, 2020, no. 4 (35), pp. S. 52-56.

9. L'vovich E.M., Kholodkov A.M. Vestnik Voronezhskogo instituta vysokikh tekhnologiy, 2020, no. 3 (34), pp. 30-33.

10. L'vovich Ya.E., L'vovich I.Ya., L'vovich E.M. Vestnik Voronezhskogo instituta vysokikh tekhnologiy, 2020, no. 3 (34), pp. 27-29.

\section{ДАННЫЕ ОБ АВТОРАХ}

Львович Яков Евсеевич, доктор технических наук, профессор Федеральное государственное образовательное учреждение выстего образования Воронежский государственный технический университет

ул. 20 лет Октября, 84, г. Воронеж, 394006, Российская Федераџия Komkovvivt@yandex.ru

ORCID: 0000-0002-7051-3763 
Преображенский Андрей Петрович, доктор технических наук, доцент

Автономная некоммерческая образовательная организащия высшего образования Воронежский институт высоких технологий ул. Ленина, 73a, г. Воронеж, 394043, Российская Федераиия Komkovvivt@yandex.ru

ORCID: 0000-0002-6911-8053

Преображенский Юрий Петрович, кандидат технических наук, доцент

Автономная некоммерческая образовательная организация высшего образования Воронежский институт высоких технологий ул. Ленина, 73a, г. Воронеж, 394043, Российская Федерация Komkovvivt@yandex.ru

\section{DATA ABOUT THE AUTHORS}

Yakov E. Lvovich, Doctor of Technical Sciences, Professor Voronezh State Technical University 84, 20 years of October Str., Voronezh, 394006, Russian Federation Komkovvivt@yandex.ru

ORCID: 0000-0002-7051-3763

Andrey P. Preobrazhenskiy, Doctor of Technical Sciences, Associate Professor Voronezh Institute of High Technologies 73a, Lenin Str., Voronezh, 394043, Russian Federation Komkovvivt@yandex.ru ORCID: 0000-0002-6911-8053

Yuriy P. Preobrazhenskiy, Candidate of Technical Sciences, Associate Professor Voronezh Institute of High Technologies 73a, Lenin Str., Voronezh, 394043, Russian Federation Komkovvivt@yandex.ru 